\title{
When does a semiring become a residuated lattice?*
}

\author{
Ivan Chajda and Helmut Länger
}

\begin{abstract}
It is an easy observation that every residuated lattice is in fact a semiring because multiplication distributes over join and the other axioms of a semiring are satisfied trivially. This semiring is commutative, idempotent and simple. The natural question arises if the converse assertion is also true. We show that the conversion is possible provided the given semiring is, moreover, completely distributive. We characterize semirings associated to complete residuated lattices satisfying the double negation law where the assumption of complete distributivity can be omitted. A similar result is obtained for idempotent residuated lattices.
\end{abstract}

AMS Subject Classification: 06B23, 06B99, 16Y60

Keywords: semiring, DNL-semiring, completely distributive semiring, residuated lattice, double negation law

It is well-known that semirings are useful structures with applications both in mathematics and in computer science, see e.g. [3] for details. In particular, unitary rings as well as bounded distributive lattices are examples of semirings. We recall the definition of a semiring from the monograph by J. S. Golan ([2]).

Definition 1. A semiring is an algebra $\mathcal{S}=(S,+, \cdot, 0,1)$ of type $(2,2,0,0)$ satisfying the following conditions for all $x, y, z \in S$ :

(i) $(S,+, 0)$ is a commutative monoid,

(ii) $(S, \cdot, 1)$ is a monoid,

(iii) $(x+y) \cdot z=x \cdot z+y \cdot z$ and $x \cdot(y+z)=x \cdot y+x \cdot z$,

(iv) $x \cdot 0=0 \cdot x=0$.

$\mathcal{S}$ is called

- idempotent if it satisfies the identity $x+x \approx x$ (i.e., $(S,+)$ is a semilattice),

\footnotetext{
${ }^{1}$ Support of the research by the bilateral project "New perspectives on residuated posets", supported by the Austrian Science Fund (FWF), project I 1923-N25, and the Czech Science Foundation (GAČ), project 15-34697L, as well as by AKTION Austria - Czech Republic, project 75p11, is gratefully acknowledged.

*Preprint of an article published in Asian-European Journal of Mathematics, Vol. 9, No. 4, 2016, pages 1650088-1-1650088-10, DOI: 10.1142/S1793557116500881 C) World Scientific Publishing Company, https : //www. worldscientific.com/worldscinet/aejm
} 
- commutative if it satisfies the identity $x \cdot y \approx y \cdot x$,

- simple (see e.g. [2]) if it satisfies the identity $x+1 \approx 1$.

Recall that a $\operatorname{ring} \mathcal{R}=(R,+, \cdot)$ is called Boolean if it satisfies the identity $x \cdot x=x$. Note that any Boolean ring is commutative and of characteristic 2. If $\mathcal{R}$ is a Boolean ring with 1 then it can be organized into a Boolean algebra. Also conversely, every Boolean algebra can be organized into a Boolean ring with 1. Since Boolean algebras form an algebraic axiomatization of the classical propositional logic, we can ask if a similar role is played by semirings for certain non-classical logics. The answer is partly positive. The aim of this paper is to study this question.

For the concept of a residuated lattice we use the definition given in [1].

Definition 2. A residuated lattice is an algebra $\mathcal{L}=(L, \vee, \wedge, \odot, \rightarrow, 0,1)$ of type $(2,2,2$, $2,0,0)$ satisfying the following conditions for all $x, y, z \in L$ :

(i) $(L, \vee, \wedge, 0,1)$ is a bounded lattice,

(ii) $(L, \odot, 1)$ is a commutative monoid,

(iii) $x \odot y \leq z$ if and only if $x \leq y \rightarrow z$.

Here $\leq$ denotes the lattice order. Condition (iii) is called the adjointness property. The operation $\rightarrow$ is called residuum and for $a, b \in L, a \rightarrow b$ is called the residuum of $b$ by $a$.

We recall some well-known facts concerning residuated lattices:

Lemma 3. Let $\mathcal{L}=(L, \vee, \wedge, \odot, \rightarrow, 0,1)$ be a residuated lattice and $a, b \in L$. Then (i) (vi) hold:

(i) $a \rightarrow b$ is the greatest element of $\{x \in L \mid x \odot a \leq b\}$,

(ii) $\neg 0=1$,

(iii) $\neg 1=0$,

(iv) $a \odot \neg a=0$,

(v) $a \leq \neg(\neg a)$,

(vi) $a \leq b$ implies $\neg b \leq \neg a$.

Here and in the following $\neg a$ is an abbreviation for $a \rightarrow 0$ and is called the negation of a. Note that $\neg a$ is the greatest element of $\{x \in L \mid x \odot a=0\}$.

Proof. Follows easily from the definition. See e.g. Theorems 2.17 and 2.30 of [1].

Recall that residuated lattices form an algebraic axiomatization of fuzzy logics, see [1] for details and for the complete explanation. Hence, when studying connections between semirings and residuated lattices, we in fact ask for an algebraic axiomatization of fuzzy logics by means of certain semirings in the way known for classical logic and Boolean rings. 
Theorem 4. Let $\mathcal{L}=(L, \vee, \wedge, \odot, \rightarrow, 0,1)$ be a residuated lattice. Then

$$
\mathbf{S}(\mathcal{L}):=(L, \vee, \odot, 0,1)
$$

is a commutative idempotent simple semiring.

Proof. Let $a, b, c \in L$. Since $(L, \vee, \wedge, 0,1)$ is a bounded lattice, its reduct $(L, \vee, 0)$ is a commutative monoid. Of course, $\vee$ is idempotent. Further, $(L, \odot, 1)$ is a commutative monoid by definition. Since 0 is the least element of $\mathcal{L}$, we have $0 \leq a \rightarrow 0$ and according to the adjointness property $0 \odot a \leq 0$ and thus $0 \odot a=0$. The law

$$
a \odot(b \vee c)=(a \odot b) \vee(a \odot c)
$$

holds according to Theorem 2.25 of [1]. Finally, $a \vee 1=1$. Altogether, $\mathbf{S}(\mathcal{L})=$ $(L, \vee, \odot, 0,1)$ is a commutative idempotent simple semiring.

Remark 5. Theorem 2.25 of [1] reads as follows:

(distributivity of $\odot, \rightarrow$ over $\wedge, \vee$ ) The following are true for each index set $I$. Moreover, in the first three formulas, if the left side makes sense, then so does the right side.

$$
\begin{aligned}
x \odot \bigvee_{i \in I} y_{i} & =\bigvee_{i \in I}\left(x \odot y_{i}\right), \\
x \rightarrow \bigwedge_{i \in I} y_{i} & =\bigwedge_{i \in I}\left(x \rightarrow y_{i}\right), \\
\bigvee_{i \in I} x_{i} \rightarrow y & =\bigwedge_{i \in I}\left(x_{i} \rightarrow y\right), \\
x \odot \bigwedge_{i \in I} y_{i} & \leq \bigwedge_{i \in I}\left(x \odot y_{i}\right), \\
\bigvee_{i \in I}\left(x \rightarrow y_{i}\right) & \leq x \rightarrow \bigvee_{i \in I} y_{i}, \\
\bigvee_{i \in I}\left(x_{i} \rightarrow y\right) & \leq \bigwedge_{i \in I} x_{i} \rightarrow y .
\end{aligned}
$$

We want to investigate if the converse statement also holds. At first we need the following

Lemma 6. Let $\mathcal{S}=(S,+, \cdot, 0,1)$ be an idempotent semiring and $\leq$ the induced order of the join-semilattice $(S,+)$. Then $\cdot$ is isotone, i.e., $a \leq b$ implies both $a \cdot c \leq b \cdot c$ and $c \cdot a \leq c \cdot b$, for all $a, b, c \in S$.

Proof. Assume $a \leq b$. Using distributivity of - with respect to + we have

$$
a \cdot c \leq a \cdot c+b \cdot c=(a+b) \cdot c=b \cdot c .
$$

The second inequality can be shown analogously.

Let $(S,+)$ be a join-semilattice and $x_{i} \in S$ for all $i \in I$. Then the sum $\sum_{i \in I} x_{i}$ denotes the supremum of the $x_{i}$ (if it exists). Of course, $\sum_{i \in I} x_{i}$ exists in case $I$ is finite.

In order to obtain a residuated lattice from a given semiring we need the following concept. 
Definition 7. A commutative idempotent semiring $\mathcal{S}=(S,+, \cdot, 0,1)$ is called completely distributive if the following conditions hold:

(i) In the join-semilattice $(S,+)$, the sum $\sum_{i \in I} y_{i}$ exists for all sets $I$ and $y_{i} \in S(i \in I)$,

(ii) $x \cdot\left(\sum_{i \in I} y_{i}\right)=\sum_{i \in I}\left(x \cdot y_{i}\right)$ for all $x, y_{i} \in S(i \in I)$.

Note that both conditions hold in case $S$ is finite.

Now we can prove the following result.

Theorem 8. Let $\mathcal{S}=(S,+, \cdot, 0,1)$ be a commutative idempotent simple completely distributive semiring. Then there exist binary operations $\wedge$ and $\rightarrow$ on $S$ such that

$$
\mathbf{L}(\mathcal{S}):=(S,+, \wedge, \cdot, \rightarrow, 0,1)
$$

is a residuated lattice.

Proof. Clearly, $(S,+, 0)$ is a join-semilattice with least element 0 . Let $a, b, c \in S$. Because of the simplicity of $\mathcal{S}$ we have $a \leq 1$ where $\leq$ denotes the induced semilattice order. In other words, $(S,+, 0,1)$ is a bounded join-semilattice. According to (i) of Definition 7 , in the poset $(S, \leq)$ there exist arbitrary suprema and hence also arbitrary infima. Therefore we obtain a bounded complete lattice $(S,+, \wedge, 0,1)$. Put

$$
a \rightarrow b:=\sum_{a \cdot x \leq b} x
$$

From this follows that $a \cdot b \leq c$ implies $a \leq b \rightarrow c$. Conversely, according to complete distributivity of $\mathcal{S}$ and isotonicity of $\cdot$, the assertion $a \leq b \rightarrow c$ implies

$$
a \cdot b \leq(b \rightarrow c) \cdot b=\left(\sum_{b \cdot x \leq c} x\right) \cdot b=\sum_{b \cdot x \leq c}(x \cdot b) \leq c .
$$

This completes the proof of the theorem.

Corollary 9. Let $\mathcal{S}=(S,+, \cdot, 0,1)$ be a finite commutative idempotent simple semiring. Then there exist binary operations $\wedge$ and $\rightarrow$ on $S$ such that

$$
\mathbf{L}(\mathcal{S}):=(S,+, \wedge, \cdot, \rightarrow, 0,1)
$$

is a residuated lattice.

Remark 10. By the previous theorem we see that complete distributivity of commutative idempotent simple semirings implies the adjointness property and, conversely, the adjointness property implies distributivity of the derived semiring (as shown e.g. in Theorem 2.25 in [1]).

When studying residuated lattices, we usually ask for some additional conditions that are satisfied by algebras derived from residuated lattices. One of them is the so-called double negation law. 
Definition 11. A residuated lattice $\mathcal{L}=(L, \vee, \wedge, \odot, \rightarrow, 0,1)$ is said to satisfy the double negation law if it satisfies the identity $\neg(\neg x)=x$.

For example, an MV-algebra is a residuated lattice satisfying the double negation law as well as the so-called divisibility, i.e. the identity $x \wedge y \approx x \odot(x \rightarrow y)$ (cf. Theorem 2.42 of [1]).

For the reader's convenience, we recall the definition of an MV-algebra. An MV-algebra is an algebra $(L, \oplus, \neg, 0)$ of type $(2,1,0)$ satisfying the following identities:

$$
\begin{aligned}
(x \oplus y) \oplus z & \approx x \oplus(y \oplus z), \\
x \oplus y & \approx y \oplus x, \\
x \oplus 0 & \approx x, \\
x \oplus \neg 0 & \approx \neg 0, \\
\neg(\neg x) & \approx x, \\
\neg(\neg x \oplus y) \oplus y & \approx \neg(\neg y \oplus x) \oplus x .
\end{aligned}
$$

If one defines

$$
\begin{aligned}
x \rightarrow y & :=\neg x \oplus y, \\
x \vee y & :=(x \rightarrow y) \rightarrow y, \\
x \wedge y & :=\neg(\neg x \vee \neg y), \\
x \odot y & :=\neg(\neg x \oplus \neg y), \\
1 & :=\neg 0
\end{aligned}
$$

then $(L, \vee, \wedge, \odot, \rightarrow, 0,1)$ is a residuated lattice.

As pointed out in Theorem 8 , if $\mathcal{S}=(S,+, \cdot, 0,1)$ is a commutative idempotent simple semiring then $(S,+, 0,1)$ is a bounded join-semilattice. Let again denote the induced order by $\leq$ and assume that every subset of $S$ has a supremum with respect to $\leq$. Then $(S, \leq)$ is a complete lattice and we can define a unary operation $n$ on $S$ as follows:

$$
n(x):=\sum_{x \cdot y=0} y \text { for all } x \in S
$$

The following result is an easy consequence of the definition of $n$.

Lemma 12. If $\mathcal{S}=(S,+, \cdot, 0,1)$ is a commutative idempotent simple completely distributive semiring and $a \in S$ then $a \cdot n(a)=0$ and $a \leq n(n(a))$.

Proof. We have

$$
a \cdot n(a)=a \cdot \sum_{a \cdot y=0} y=\sum_{a \cdot y=0}(a \cdot y)=0
$$

and hence

$$
n(n(a))=\sum_{n(a) \cdot y=0} y \geq a .
$$


In what follows, we provide a correspondence between complete residuated lattices satisfying the double negation law and a certain class of semirings. Contrary to Theorem 8 we do not need complete distributivity in this case.

Definition 13. A DNL-semiring (double negation law semiring) is a commutative idempotent simple semiring $\mathcal{S}=(S,+, \cdot, 0,1)$ such that $(S,+)$ is a complete join-semilattice and the following identities are satisfied for the function $n$ defined by (1):

(i) $n(n(x)) \approx x$

(ii) $x \cdot n(x \cdot n(y)) \leq y($ i.e., $x \cdot n(x \cdot n(y))+y \approx y)$,

(iii) $x \cdot n(x+y) \approx 0$.

We are now able to prove the following result:

Theorem 14. Let $\mathcal{S}=(S,+, \cdot, 0,1)$ be a DNL-semiring and put

$$
\begin{aligned}
x \wedge y & :=n(n(x)+n(y)) \text { and } \\
x \rightarrow y & :=n(x \cdot n(y))
\end{aligned}
$$

for all $x, y \in S$. Then

$$
\mathbf{L}(\mathcal{S}):=(S,+, \wedge, \cdot, \rightarrow, 0,1)
$$

is a complete residuated lattice satisfying the double negation law.

Proof. Let $a, b, c \in S$. Since $\mathcal{S}=(S,+, \cdot, 0,1)$ is a DNL-semiring, $(S, \cdot, 1)$ is a commutative monoid and $(S, \leq)$ a complete lattice with smallest element 0 and greatest element 1 . Here $\leq$ denotes the induced order of $\mathcal{S}$. First of all we have to show that $n(n(x)+n(y))$ is the infimum of $x$ and $y$ with respect to $\leq$. According to (i) of Definition [13, $n$ is an involution. If $a \leq b$ then

$$
n(b)=\sum_{b \cdot y=0} y \leq \sum_{a \cdot y=0} y=n(a)
$$

since according to Lemma 6 we have for all $y \in S$ that $b \cdot y=0$ implies $a \cdot y=0$. Since $n$ is an involution we have $a \leq b$ if and only if $n(b) \leq n(a)$, i.e., $n$ is an order isomorphism from $(S, \leq)$ to $(S, \geq)$. Let $x \wedge y$ denote the infimum of $x$ and $y$ with respect to $\leq$. Then $n(x \wedge y)=n(x)+n(y)$ and hence $x \wedge y=n(n(x)+n(y))$. Next we want to prove the double negation law. Put

$$
\neg a:=a \rightarrow 0 .
$$

Then

$$
\neg a=n(a \cdot n(0))=n(a \cdot 1)=n(a)
$$

and according to (i) of Definition 13 we have $\neg(\neg a)=a$ proving the double negation law. Finally, we need to prove the adjointness property. If $a \leq b$ then according to (iii) of Definition 13 we have

$$
a \rightarrow b=n(a \cdot n(b))=n(a \cdot n(a+b))=n(0)=1 .
$$

The first equality holds according to the definition of $\rightarrow$.

Conversely, if $a \rightarrow b=1$ then $n(a \cdot n(b))=1$ and according to (ii) of Definition 13

$$
a=a \cdot 1=a \cdot n(a \cdot n(b)) \leq b .
$$


Hence

$$
a \leq b \text { if and only if } a \rightarrow b=1 .
$$

Using this equivalence and (i) of Definition 13 we see that the following are equivalent:

$$
\begin{aligned}
a \cdot b & \leq c \\
a \cdot b \rightarrow c & =1, \\
n(a \cdot b \cdot n(c)) & =1, \\
n(a \cdot n(n(b \cdot n(c)))) & =1, \\
a \rightarrow n(b \cdot n(c)) & =1, \\
a & \leq n(b \cdot n(c)), \\
a & \leq b \rightarrow c .
\end{aligned}
$$

This shows the adjointness property. Altogether, $\mathbf{L}(\mathcal{S})=(S,+, \wedge, \cdot, \rightarrow, 0,1)$ is a complete residuated lattice satisfying the double negation law.

Theorem 15. Let $\mathcal{L}=(L, \vee, \wedge, \odot, \rightarrow, 0,1)$ be a complete residuated lattice satisfying the double negation law. Then

$$
\mathbf{S}(\mathcal{L}):=(L, \vee, \odot, 0,1)
$$

is a DNL-semiring.

Proof. Let $a, b, c \in L$. According to Theorem 4, $\mathbf{S}(\mathcal{L})$ is a commutative idempotent simple semiring. According to Theorem 2.40 in [1], $a \rightarrow b=\neg(a \odot \neg b)$ since $\mathcal{L}$ satisfies the double negation law. Hence, according to Theorem 2.17 in [1] we have

$$
\neg a=a \rightarrow 0=\bigvee_{a \cdot x=0} x=n(a) .
$$

Thus $n(n(a))=\neg(\neg a)=a$ proving (i) of Definition 13. According to Theorem 2.18 in [1], every residuated lattice satisfies the following identities:

(a) $x \odot(x \rightarrow y) \leq y$ (i.e., $(x \odot(x \rightarrow y)) \vee y \approx y)$,

(b) $x \rightarrow(x \vee y) \approx 1$.

Identity (a) can be rewritten as

$$
x \odot \neg(x \odot \neg y) \leq y
$$

which is (ii) of Definition 13, Moreover, identity (b) can be rewritten as

$$
\neg(x \odot \neg(x \vee y)) \approx 1 .
$$

Thus, according to the double negation law

$$
x \odot \neg(x \vee y) \approx 0
$$

which is (iii) of Definition 13. Hence $\mathbf{S}(\mathcal{L})$ is a DNL-semiring.

We can prove that the correspondence described in the last two theorems is one-to-one. 


\section{Theorem 16.}

(i) If $\mathcal{S}=(S,+, \cdot, 0,1)$ is a DNL-semiring then $\mathbf{S}(\mathbf{L}(\mathcal{S}))=\mathcal{S}$.

(ii) If $\mathcal{L}=(L, \vee, \wedge, \odot, \rightarrow, 0,1)$ is a complete residuated lattice satisfying the double negation law then $\mathbf{L}(\mathbf{S}(\mathcal{L}))=\mathcal{L}$.

Proof.

(i) If $\mathbf{L}(\mathcal{S})=(S,+, \wedge, \cdot, \rightarrow, 0,1)$ then $\mathbf{S}(\mathbf{L}(\mathcal{S}))=(S,+, \cdot, 0,1)=\mathcal{S}$.

(ii) We have $\mathbf{S}(\mathcal{L})=(L, \vee, \odot, 0,1)$. Put $\mathbf{L}(\mathbf{S}(\mathcal{L}))=(L, \vee, \cap, \odot, \Rightarrow, 0,1)$ and let $a, b \in L$. Since the orders in $\mathcal{L}$ and $\mathbf{L}(\mathbf{S}(\mathcal{L}))$ coincide we have $a \cap b=a \wedge b$. Furthermore,

$$
\begin{aligned}
& n(a)=\bigvee_{a \odot x=0} x=\bigvee_{a \odot x \leq 0} x=a \rightarrow 0=\neg a \text { and } \\
& a \Rightarrow b=n(a \odot n(b))=\neg(a \odot \neg b)=a \rightarrow b .
\end{aligned}
$$

Hence $\mathbf{L}(\mathbf{S}(\mathcal{L}))=\mathcal{L}$.

Another useful property of residuated lattices is idempotency. A residuated lattice $\mathcal{L}=$ $(L, \vee, \wedge, \odot, \rightarrow, 0,1)$ is called idempotent if it satisfies the identity $x \odot x=x$.

Theorem 17. A residuated lattice $\mathcal{L}=(L, \vee, \wedge, \odot, \rightarrow, 0,1)$ is idempotent if and only if $\mathbf{S}(\mathcal{L})$ is a bounded distributive lattice.

Proof. Let $a, b \in L$. According to Theorem 2.17 in [1] we have $a \odot b \leq a \wedge b$. Since the operation $\odot$ is monotone (Theorem 2.23 in [1]) and $a \wedge b \leq a$ and $a \wedge b \leq b$, using idempotency we infer

$$
a \wedge b=(a \wedge b) \odot(a \wedge b) \leq a \odot b \leq a \wedge b
$$

proving that $\wedge$ and $\odot$ coincide. According to distributivity ((iii) of Definition 1 and Theorem 4) we obtain that $\mathbf{S}(\mathcal{L})$ is a bounded distributive lattice. The converse assertion is trivial.

Corollary 18. If $\mathcal{L}$ additionally satisfies the double negation law then $\mathcal{L}$ is idempotent if and only if $\mathbf{S}(\mathcal{L})$ is a Boolean algebra.

Proof. This follows from the previous proof, from (iv) and (vi) of Lemma 3 and from the double negation law.

Definition 19. A residuated lattice $(L, \vee, \wedge, \odot, \rightarrow, 0,1)$ is called prelinear if it satisfies the identity

$$
(x \rightarrow y) \vee(y \rightarrow x) \approx 1 .
$$

Corollary 20. For a semiring $\mathcal{S}=(S,+, \cdot, 0,1)$ the following are equivalent: 
(i) $\mathcal{S}$ is a DNL-semiring satisfying the identity $x \cdot n(n(y)+n(z)) \approx n(n(x \cdot y)+n(x \cdot z))$.

(ii) $\mathbf{L}(\mathcal{S})=(S, \vee, \wedge, \cdot, \rightarrow, 0,1)$ is a complete and prelinear residuated lattice satisfying the double negation law where $x \vee y=x+y, x \wedge y=n(n(x)+n(y)), x \odot y=x \cdot y$ and $x \rightarrow y=n(x \cdot n(y))$ for all $x, y \in S$.

Proof. According to Theorems 14, 15 and 16, $\mathcal{S}$ is a DNL-semiring if and only if $\mathbf{L}(\mathcal{S})$ is a complete residuated lattice satisfying the double negation law. Because of Theorem 2.40 in [1, $\mathbf{L}(\mathcal{S})$ is prelinear if and only if it satisfies the identity

$$
x \cdot(y \wedge z) \approx(x \cdot y) \wedge(x \cdot z)
$$

which is equivalent to

$$
x \cdot n(n(y)+n(z)) \approx n(n(x \cdot y)+n(x \cdot z)) .
$$

\section{References}

[1] R. Bělohlávek, Fuzzy Relational Systems. Foundations and Principles. Kluwer, New York 2002. ISBN 0-306-46777-1.

[2] J. S. Golan, The Theory of Semirings with Applications in Mathematics and Theoretical Computer Science. Longman, Harlow 1992. ISBN 0-582-07855-5.

[3] W. Kuich and A. Salomaa, Semirings, Automata, Languages. Springer, Berlin 1986. ISBN 3-540-13716-5.

Authors' addresses:

Ivan Chajda

Palacký University Olomouc

Faculty of Science

Department of Algebra and Geometry

17. listopadu 12

77146 Olomouc

Czech Republic

ivan.chajda@upol.cz

Helmut Länger

TU Wien

Faculty of Mathematics and Geoinformation

Institute of Discrete Mathematics and Geometry

Wiedner Hauptstraße 8-10

1040 Vienna

Austria

helmut.laenger@tuwien.ac.at 